\title{
Case Study: Vertebrobasilar insufficiency imitating cerebrovasculitis in a patient with systemic lupus erythematosus
}

\section{Mariela Geneva-Popova, Stanislava Popova}

Medical University of Ploviv, Department of Propedeutic of Internal Diseases, Clinic of Rheumatology, UMHAT "Sv. Georgi”, Plovdiv, Bulgaria

\section{Case Presentation}

History of Present Illness: We report a 45-year-old patient, who treated at the Rheumatology Clinic, "St. George" Hospital, Plovdiv, Bulgaria with excruciating pain, increasing in the night hours, headache for about a week. The pain is accompanied by nausea, restlessness, suicidal thoughts, crying. 2011 systemic lupus erythematosus was found in the patient. Disease debuted with nephrotic syndrome, hematological and immunological abnormalities. Treatment with megadoses corticosteroid 3x1000 mg and cyclophosphamide $500 \mathrm{mg}$ monthly was started from 2011 to 2015. Maintenance treatment is usually $10 \mathrm{mg}$ prednisolone, $50 \mathrm{mg}$ imuran, antihypertensive drugs.

Social History: the patient reported using cigarettes for 9 packs of years; she sowed quitting smoking six years ago. She denies the use of alcohol and illegal drugs. She is not married. He works as a shop assistant and has a meager social life.

Allergies: No known medicine, food, or environmental allergies. 
Past Medical History: Arterial hypertension

Past Surgical History: there is no

Medications: Prednisolon $10 \mathrm{mg}$ by mouth every day, Imuran 50 by mouth every day, Perindopril $1010 \mathrm{mg}$ by mouth every day

Physical Exam: Vitals: Temperature, 97.8 F; heart rate 78; respiratory rate, 16; blood pressure 130/86; body mass index, 26

General: She is good looking, but worried, lying down due to a severe headache $\mathbf{R}$

espiratory: She has vesicular breathing, no wheezing and friction,

Cardiovascular: She has a regular rate and rhythm with no murmurs, rubs, or gallops.

Succusio renalis was negative bilaterally

Gastrointestinal: Bowel sounds X4. No bruits or pulsatile mass, no peripheral edema was found.

\section{Initial Evaluation}

Laboratory Studies were in reference values. Immunological tests were completely negative as they did not support the thesis of disease activity, as follows urine - specific gravity: 1.007, urine $\mathrm{pH}$ : 8.0 , protein - qualitative and semi-quantitative: (+/-) traces, glucose - qualitative and semi-quantitative: (-) negative, urobilinogen - qualitative and semi-quantitative: not increased / - /, bilirubin - qualitative and semi-quantitative: (-) neg.; erys - sediment: 1-2 erhyt., 2-3 lks. Hemoglobin $124.0 \mathrm{~g} / \mathrm{l}$; erythrocytes 4.64.10 12 ; Hematocrit 0.337 1/1; Mean erythrocyte volume (MCV): $72.6 \mathrm{fl}$; MCH index: $22.3 \mathrm{pg}$; MCHC index: $308.0 \mathrm{~g} / \mathrm{l}$; RDW: 19.0\%; Leukocytes (WBC): 4.62 10\%/1; Platelets (PLT): 267.0.10 9 /1; Mean platelet volume (MPV): 10.0 fl; Platelet (PCT): 0.267\%; PDW: 13.1\%; St)/ - /\%; Neutrophil granulocytes (Sg): 3.93.10. ${ }^{9} / 1$; Neutrophil granulocytes (Sg): 84.9\%; Eosinophils (EOS): 0.04.10 $/ 1$; Eosinophils 0.8\%; Basophils 0.01.10 $/ 1$; Basophils 0.2\%; Monocytes: 0.15.10 $/ 1$; Monocytes 3.3\%; Lymphocytes 0.49.10\%/1; Lymphocytes 10.8\%; Westergren (ESR): $30 \mathrm{~mm} / \mathrm{h}$; Prothrombin time 26.4\%; Prothrombin time, INR: 1.89, Prothrombin time, sec: 20.6 sec; DFibr: / - /; Activated partial thromboplastin time (APTT): $31.3 \mathrm{sec}$; Fibrinogen (Fbg): 4.96g/1; glucose $3.7 \mathrm{mmol} / \mathrm{l}$; Creatinine - serum: $80.0 \mathrm{mlmol} / \mathrm{L}$; Uric acid - serum: $225.0 \mu \mathrm{mol} / \mathrm{L}$; Urea serum: 
4.0mmol/1; Complement C3 0.81 g/l; Complement C4 0.38 g/l; AST 14.9 U/ 1; ALT: 10 1U/1; Creatine kinase 61.0 U/l, Protein -U-CSF PROT: 0.052 g/24h.

Immunological tests: Antinuclear antibodies (ANA IgG) - IFA - 1: 160 negative /-/ (normal (below 1: 160), anti-ds-DNA (Ig M, G, A) - 76 (norm 100 IU / ml), anti-Sm antibodies (Ig M, G, A) - 5.34 (norm $25 \mathrm{IU} / \mathrm{ml}$ ), anticardiolipins antibodies Ig M, G 8,61 (norm 12 GPL/U/ml), anticardiolipins antibodies Ig G, G 12.45 (norm 12 MPL / U / ml), antineutrophilcytoplasmic-ANCA-MPO (IgG, M, A) - ELISA - <20 Ru / ml (norm 20 IU/ml), antineutrophil-cytoplasmic-ANCA-PR3 (IgG, M, A - ELISA - <20 Ru / ml, (norm $20 \mathrm{IU} / \mathrm{ml}$ ), rheumatoid factor (RF) (IgM) - ELISA - 16.2 IU / ml (norm $20 \mathrm{IU} / \mathrm{ml}$ ), anti-CCP (IgG) ELISA - $<5 \mathrm{Ru} / \mathrm{ml}$ (norm 25) Ru / ml, detection of hepatitis B virus surface antigen (HBV) HBsAg by ELISA - (-) negative; detection of markers of hepatitis C virus (HCV) - anti-HCV or HCV Ag / Ab by ELISA - (-) negative, detection of antibodies against HIV 1/2 or HIV Ag / Ab by ELISA: (-) negative.

\section{Differential Diagnosis}

- Neurolupus

- Spatially occupying process of the central nervous system

- Cerebral infarction

- Antiphospholipid syndrome

- Drug intoxication

- Demyelinating process

- Accelerated atherosclerosis

- Chronic fatigue syndrome

- $\quad$ Lyme disease.

- Mixed cryoglobulinemia within hepatitis Chepatitis

- Polyarteritis

- Vasculitis

- $\quad$ Serum disease.

- Thrombotic thrombocytopenic purpura. 


\section{Confirmatory Evaluation}

During the stay in the clinic, the general condition of the patient deteriorated, and despite the use of conventional drugs for the treatment of SLE - high-dose corticosteroids and cytostatics, the condition did not improve. From the 4th day onwards, the patient had twice periods of hallucinations, due to which it was necessary to consult a neurologist, ophthalmologist, psychiatrist. The conclusion of the neurologist is the presence of hypertensive encephalopathies, with an ophthalmologist - without evidence of papillary edema, with a psychiatrist - with evidence of neurolupus.

As diagnostic procedures to the patient's usual lumbar puncture was performed - which was negative, CT of the brain - conclusion - without evidence of fresh ischemic or hemorrhagic lesions, ventricular system and subarachnoid spaces normal. From MRI results - a filamentous narrowing of the lumen of the left vertebral artery was found, which may be associated with vertebrobasilar insufficiency. The remaining cerebral vessels have an unchanged lumen, with no evidence of stenosis or aneurysmal dilatation (Fig.1). 


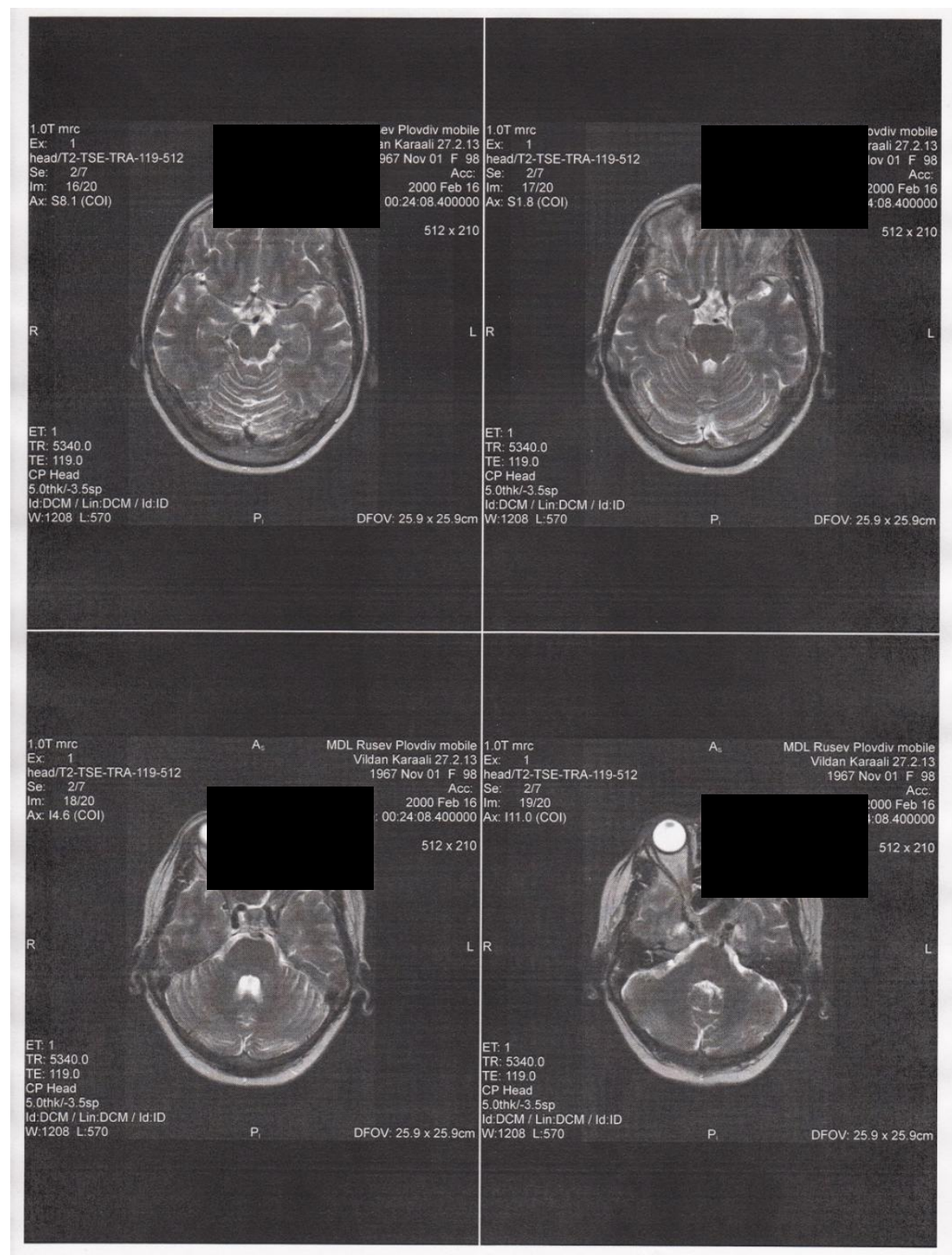

Fig.1. MRI of the brain, which does not confirm the presence of cerebrovasculitis, the study confirmed the stenosis of the basal artery

The results of immunological and imaging studies proved low morbidity of the underlying disease, lack of MRI criteria for cerebrovasculitis and significant basal artery stenosis.

\section{Diagnosis}

The results of immunological and imaging studies conducted low morbidity of the underlying disease, lack of MRI criteria for cerebrovasculitis and significant basal artery stenosis. 


\section{Management}

The applied treatment with a high dose of cytostatics did not affect the patient's condition. Subjective complaints decreased after the inclusion of vasodilators and statins.

\section{Discussion}

Systemic lupus erythematosus is a polysystemic, autoimmune disease characterized by inflammation of connective tissue and immunocomplex vasculitis as a result of genetic predisposition and impaired immune regulation $(1,2,3)$. This is an autoimmune disease in which the antigen-antibody reaction and subsequent immune inflammation occurs in the vessels and almost all organs and systems (vessels, skin, subcutaneous tissue, joints, lungs, brain, heart, blood tissue, etc. $(4,5)$. SLE includes: skin changes - the manifestations of the skin and visible mucous membranes, together with those of the musculoskeletal system are most often affected first by the disease in the form of "butterfly erythema", photosensitization, discoid, annular or papulosquamous lesions, aphthous ulcers, alopecia, vasculitis, perforation of the soft palate, paniculitis, etc., pulmonary manifestations, vasculitis, cardiovascular changes, gastric and laboratory changes $(1,6,7,8,9)$.

Vertebrobasilar insufficiency is due to reduced blood supply to the cerebellum (4). Causes that can lead to its development are: atherosclerotic changes in the vertebral or basilar arteries, various conditions leading to lower blood pressure, the presence of osteophytes in the vertebro-basilar canal leading to compression of the arteries, thrombosis in the initial segment of the subclavian artery and etc $(2,3)$.

Vertebasilar disorders most often occur suddenly. Patients may experience severe dizziness, nausea, vomiting, loss of balance, and sometimes short-term seizures with complete recovery (10). This symptomatology is most often described as Meniere's syndrome (identical symptomatology in inner ear injuries) (7).

The detection of pathological changes in vessels requires mandatory angiographic examination. The conventional radiography of the cervical vertebrae is also part of the diagnostic algorithm, and in case of a finding, a Computed Tomography (CT) of the areas of the neck in which the established changes are found (most often osteophytes) can be performed $(3,4)$. 
Neurolupus is one of the important sites of lupus disease $(11,12)$. Along with epileptiform seizures and psychotic manifestations, which are also criteria for diagnosis, there is a significant variety of symptoms: headache, psychosis, paralysis, paresis, sensory disorders, cortical atrophy and others (3). Differential diagnosis of the disease is made with rheumatic diseases, chronic febrile conditions, diseases of various organs, which occur with immune phenomena close to those of systemic lupus erythematosus, primary phospholipid syndrome and others $(13,14,15,16,17)$.

The treatment of Systemic Lupus Erythematosus has two main aspects: hygienic-dietary regime and medical treatment. The usual agents for the treatment of SLE are antimalarials, glucocorticosteroids and immunosuppressants in megadoses (18). After 5-10 years of treatment of patients with these drugs, the side effects of taking these drugs are usually observed - more frequent and difficult to treat infections, altered immunoreactivity, changes in lipid and carbohydrate profile $(4,5,6,19)$.

Due to the natural course of neurolupus and the administration of high-dose corticosteroids and immunosuppressants in patients with neurolupus for more than 5 years, manifestations such as cerebrovascular disease or vascular dementia have been observed. Reasons for this are: acute ischemic disorders of cerebral circulation due to cerebrovasculitis - strokes, or chronic insufficiency of cerebral circulation, often occurring as vertebro-basilar insufficiency $(17,20)$.

In neurolupus, there is a decline in the cognitive functions of the individual and personality changes as a result of acute or chronic disorders of cerebral blood flow caused by damage to blood vessels $(13,15)$. The pathological mechanisms of damage are basically processes of obliteration and thrombosis, as well as in frequent cases of rupture of the vascular wall due to the accumulation of atherosclerotic plaques and changes in its structure. infarcts of various sizes, which are located in structures important for the functioning of the brain, such as the basal ganglia, frontal lobes (important for volitional behavior and decision-making), the thalamus. Pathological changes were also found in the subcortical white matter of the cerebral hemispheres $(1,3,5,7)$.

At the beginning of the disease, a pseudoneurasthenic syndrome is found: weakened active attention, emotional incontinence, difficult acquisition of new knowledge and skills, difficult switching of attention, which is expressed in difficult transfer of attention from one activity to another. Against the background of the above symptoms appear: hypochondria, complaints 
of headache, which intensifies in the evening or after exercise, dizziness is often reported. Patients are prone to depression (21).

In the development of the disease, memory disorders come to the fore, which usually make a stronger impression on others, and it becomes clear that there is a real problem. Memory deficit in clear consciousness mainly affects short-term and fixation memory. In parallel, one and more often several of the following cognitive disorders appear: disorders in orientation, concentration, in the ability to plan activities, impaired ability to perform complex complex tasks, aphasia, apraxia $(22,23)$. Later, in addition to the fixation memory, the retention memory (responsible for the storage of information) and the reproduction memory (the one that is expressed in memory) are affected. Thinking slows down, becomes unnecessarily detailed, the ability to distinguish the essential from the inessential is lost $(3,5)$.

Treatment of vertebrobasilar insufficiency is effective, but requires considerable patience on the part of patients and physicians. Drugs from the group of vasodilators are used, acting mainly in the cerebral vascular bed and nootropic drugs that improve metabolic processes in neurons. In insomnia, sleeping pills can be used, and in the presence of fear tension anxiolytics. The dosing of psychotropic drugs should be done with extreme caution, as small doses of them can further affect their condition.

The prevention of vascular dementia associated with SLE and vertebral insufficiency is actually the prevention of atherosclerosis. Untreated hypertension and poor blood sugar control should not be allowed. Lipid status should be monitored regularly and, in the event of abnormalities, appropriate dietary regimens and, if necessary, medical treatment should be considered.

\section{References}

[1] Mills J., "Systemic lupus erythematosus”, N Engl J Med. 1994 Jun 30;330(26):1871-9.

[2] Sanna G., M. Bertolaccini, M. Cuadrado et al., "Neuropsychiatric manifestations in systemic lupus erythematosus: prevalence and association with antiphospholipid antibodies", $J$ Rheumatol. May 2003;30(5):985-92.

[3] Azarpazhooh M., N. Mokhber, E. Orouji et al., "Microembolic signals in patients with systemic lupus erythematosus", Can J Neurol Sci. May 2010;37(3):371-5. 
[4] Ellis S., A. Verity, "Central nervous system involvement in systemic lupus erythematosus: a review of neuropathologic findings in 57 cases, 1955-1977”, Semin Arthritis Rheum., Feb 1979;8(3):212-21.

[5] AlSaleh J., V. Jassim, M. ElSayed, et al., "Clinical and immunological manifestations in 151 SLE patients living in Dubai”, Lupus, 2008;17(1):62-6.

[6] Zanardi V., L. Magna, L. Costallat, "Cerebral atrophy related to corticotherapy in systemic lupus erythematosus (SLE)", Clin Rheumatol., 2001;20(4):245-50.

[7] Castellino G., M. Padovan, A. Bortoluzzi et al., "Single photon emission computed tomography and magnetic resonance imaging evaluation in SLE patients with and without neuropsychiatric involvement", Rheumatology (Oxford). Mar 2008;47(3): 31923.

[8] Wang H., G. Yunjie, M. Yanhong et al., “"Performance of the 2019 EULAR/ACR systemic lupus erythematosus classification criteria in a cohort of patients with biopsyconfirmed lupus nephritis", Lupus Science \& Medicine 2021;8: e000458.

[9] Lisnevskaia L., G. Murphy, D. Isenberg, Systemic lupus erythematosus”, Lancet 2014; $384: 1878-88$.

[10] Aringer M., K. Costenbader, D. Daikh et al., "European League against Rheumatism/American College of rheumatology classification criteria for systemic lupus erythematosus", Ann Rheum Dis 2019;78:1151-9.

[11] Aringer M., K. Costenbader, D. Daikh, et al., "European League against Rheumatism/American College of rheumatology classification criteria for systemic lupus erythematosus", Arthritis Rheumatol 2019;71:1400-12.

[12] Suda M., M. Kishimoto, S. Ohde et al., "Validation of the 2019 ACR/ EULAR classification criteria of systemic lupus erythematosus in 100 Japanese patients: a realworld setting analysis", Clin Rheumatol 2020;39:1823-7.

[13] Dörner T., R. Furie, "Novel paradigms in systemic lupus erythematosus", Lancet 2019; 393:2344-58.

[14] Bendorius M., Po. Chrystelle, S.Muller and H. Jeltsch-David, "From Systemic Inflammation to Neuroinflammation: The Case of Neurolupus", Int. J. Mol. Sci. 2018, 19(11), 3588. 
[15] Cohen D., E. Rijnink, R. Nabuurs et al., "Brain histopathology in patients with systemic lupus erythematosus: identification of lesions associated with clinical neuropsychiatric lupus syndromes and the role of complement”, Rheumatology (Oxford). 2017 Jan;56 (1):77-86.

[16] Mackay M., C. Tang A. Vo, “Advanced neuroimaging in neuropsychiatric systemic lupus erythematosus", Curr Opin Neurol. 2020 Jun;33(3):353-361.

[17] Appenzeller S., T. Lilian, L. Costallat, F. Cendes, "Neurolupus", Arch Neurol. 2006 Mar; $63(3): 458-60$.

[18] Chang-Youh Tsai, Ko-Jen Li, Song-Chou Hsieh et al., "What's wrong with neutrophils in lupus?”, Clin Exp Rheumatol, Jul-Aug 2019;37(4):684-693.

[19] Wieliczko M., J. Matuszkiewicz-Rowińska, "Systemic lupus erythematosus - news 2017”, Wiad Lek. 2017; 70(6 pt 2):1201-1204.

[20] Oku K., T. Atsumi, "Systemic lupus erythematosus: nothing stale her infinite variety", Mod Rheumatol., 2018 Sep;28(5):758-765.

[21] Pathak S.,C. Mohan, "Cellular and molecular pathogenesis of systemic lupus erythematosus: lessons from animal models", Arthritis Res Ther. 2011;13(5):241.

[22] Scolding N., F. Joseph, "The neuropathology and pathogenesis of systemic lupus erythematosus", Neuropathol Appl Neurobiol. 2002 Jun; 28(3):173-89.

[23] Julkunen H., “Systemic lupus erythematosus”, Duodecim. 2012;128(1):51-61. 